\title{
Preface to the special issue "Approaches for strategies of mechanical optimization in plants"
}

\author{
Taku Demura \\ Graduate School of Science and Technology, Nara Institute of Science and Technology, Ikoma, Nara 630-0192, Japan
}

As global environmental, food, and population problems get worse, various efforts for the development of a sustainable society continue worldwide. It goes without saying that plant science is expected to be an important source of innovations that would help us address these global issues. Majority of the focus on the role of plant science in developing a sustainable society has been on solving food/environmental problems by improving agricultural/forestry productivity, etc. However, it is also becoming increasingly important to develop sustainable living spaces utilizing the excellent characteristics of plants. Various initiatives have begun and the approaches in engineering of using plant biomimetics is one of several key examples that highlight the immense potential of plant inspired design and engineering philosophies (Geitmann et al. 2019; Speck and Speck 2019).

In this context, we have started a large-scale multigroup research project that aims to utilize the mechanical advantage of plants in engineering, especially in spatial structural engineering, based on the characteristics of plant cell walls, a unique structure of plants, with the support of the MEXT KAKENHI Grant-in-Aid for Scientific Research on Innovative Areas "Plant-Structure Optimization Strategy" from 2018. This project first aims to understand the mechanical properties of plants and their optimized states at multiple scales (molecular, cellular, tissue, and individual), and then to sublimate this knowledge into new structural and material models in spatial structural engineering to create the basis for the next generation of sustainable structural materials and systems.

This special issue deals mainly with the progress made in the approaches in plant biomechanics in the abovementioned research project. Plant biomechanics is extremely interdisciplinary and requires the integration of diverse research fields in plant science (from the molecular and genetic level to the individual level) with engineering, physics, mathematics, and chemistry. For this reason, readers will notice that many of articles appeared in this special issue are the result of interdisciplinary research groups.

The plant cell wall is an important key to the determination of plant biomechanics at the molecular level. It has a complex structure, consisting mainly of polysaccharides such as cellulose, hemicellulose, and pectin, and proteoglycans (Burton et al. 2010; Somerville et al. 2004). Since there is no doubt that differences in individual cell wall components affect the mechanical properties of plants, the analysis of enzymes involved in plant cell wall metabolism and cell wall peptidoglycans is fundamental to plant biomechanics at the molecular level. Many kinds of Carbohydrate-Active enZymes (CAZymes, http://www.cazy.org; Lombard et al. 2014) are known to associated with plant cell wall metabolism. Expansin is one such enzyme, and it has been suggested that they localize to the cell wall and function in the loosening of plant cell walls, however their true function remains to be elucidated (Cosgrove 2015, 2016). One reason for this is the difficulty in producing recombinant proteins heterologously (Cosgrove 2015). Matsuyama et al. (2020, pp 397-403) succeeded in improving the stability of recombinant protein of peach expansin by replacing all cysteine residues that are expected to form disulfide bonds with serine residues, thus providing a platform for further research insights into the role of these proteins. Arabinogalactan-proteins (AGPs) are extracellular proteoglycans consisting of a hydroxyproline-rich core-protein and a large portion of arabinogalactan (AG) sugar chains, called type II AGs (Seifert and Roberts 2007). Although the type II AGs are expected to function in the determination of plant cell wall properties, it is still unclear how type II AGs carry out their physiological function. Here, Ito et al. (2020, pp 459-463) examined similarities of type II AG structures from five different herbaceous plants, suggesting a conserved sugar structure among them that maybe important for its function. It is hoped that such the analysis of cell wall-related enzymes and sugar structures will provide us a major steppingstone for the elucidation of the mechanical properties of plant cell walls. 
It is obvious that the mechanical properties at the subcellular and cellular levels affect plant biomechanics. For instance, the sedimentation of intracellular amyloplasts is a key step in gravity sensing in plant gravitropism (Hagihara and Toyota 2020; Morita 2010). Interestingly, we are now able to freely manipulate the movement of intracellular organelles such as amyloplasts (Abe et al. 2020, pp 405-415): a confocal microscope equipped with optical tweezers was optimized to trap amyloplasts, which will provide us a chance to interconnect the biophysical properties of plant organelles and plant gravitropism. Measurement of the static and dynamic forces of plant cells is another important aspect for plant biomechanics at the cellular/ sub-cellular levels. Atomic force microscopy (AFM) has been used to measure the mechanical properties of plant cells (Milani et al. 2011; Peaucelle 2014), which was successfully used for the measurement of cell stiffness of Arabidopsis root cells with a newly developed system comprised of an array of glass micropillars as the support of Arabidopsis roots (Akita et al. 2020, pp 417-422). These innovations are expected to open the way to biomechanics at the cellular/sub-cellular level, and will be useful for analyzing the mechanical properties different cell wall types including those of the xylem vessel cells which has specialized cell wall patterns. While endogenous xylem vessel cells are formed deep-inside of plant bodies, a transcription factor-dependent system allows us to induce xylem vessel cell differentiation on the surface of plant bodies (Kubo et al. 2005; Yamaguchi et al. 2010). It is interesting to know what external factors (including mechanical stimuli) affect the cell wall structure of xylem vessel cells formed in this system: here, it was shown that osmotic condition, one of the mechanical stimuli, affects the cell wall pattern of xylem vessel cells, providing further insight into cellular mechanisms controlling cell wall patterning (Kunieda et al. 2020, pp 465-469).

Mechanical stimuli affect the entire individual as a result of responses at the cellular level: static loads due to its own weight and dynamic forces due to wind are good examples of this, and the plant body changes its morphology in response to them (Moulia et al. 2011; Niklas and Spatz 2000). Beier et al. (2020, pp 423-428) showed that mechanical stimuli caused by unidirectional vibrations influences an adaptive root system architecture in poplar trees. The root system has a complex threedimensional structure, which may contribute to its role as a mechanical anchor. X-ray computed tomography is effective in visualizing such the complex root system structures: with a combination of Toyoura sand, which is commercially available with well characterized physical properties, a simple method for visualizing plant roots grown in sand has been established (Yoshida et al. 2020, pp 481-484).
This special issue contains three more techniques for visualization of plant structure. A framework to capture and analyze three-dimensional (3D) structure of pavement cells of Arabidopsis cotyledons over time (four-dimensional, 4D) was developed for confocal microscopy (Higaki and Mizuno 2020, pp 429-435). This system will be useful for generating and testing theoretical models for mechanical optimization of plant cellular structures. Magnetic resonance imaging (MRI) has also been adopted to visualize plant structure: a recently developed small-bore MRI signal receiver coil was successfully used to acquire high-resolution continuous 3D structure data of pea sprouts during gravitropism (Nakai et al. 2020, pp 437-442). To capture the exact movement of plant organs, the establishment of a micro-marking technique would be a useful tool. A micro-marking technique with a nanosecond pulse laser was established, which allowed us to capture highly detailed information of pea stem bending during gravitropism (Watanabe et al. 2020, pp 485-488). To study the gravitropism with such detailed imaging data, theoretical approaches have been used (Chelakkot and Mahadevan 2017). However, methods to reconcile data and theory have yet to be established. Tsugawa et al. (2020, pp 443-450) proposed a combined data-model framework using a recently developed kinematic tool KymoRod (Bastien et al. 2016) and the elastic spring model to gain a quantitative understanding of shoot gravitropism.

In plant biomechanics, systems to measure mechanical properties of plant organs (hardness, strength of bonds between organs, forces generated by organs, etc.) are indispensable techniques. Recently, a quick method based on natural frequency (as determined from freevibration test) for evaluating stiffness of Arabidopsis inflorescence stems was proposed (Nakata et al. 2018), accuracy of which was confirmed by comparing the data of free-vibration test with the data of conventional threepoint bending tests (Nakata et al. 2020, pp 471-474). Binding capacity between organs is important issue of plant biomechanics. Notaguchi et al. (2020) has reported that the grafting ability between phylogenetically distant plant species is enhanced by a cell wall-related CAZyme, $\beta$-1,4-glucanase/GH9B3. To measure the effect of the enzyme on the mechanical properties during grafting, Kawakatsu et al. (2020, pp 451-458) established a quantitative method with a force gauge, which is expected to find utilization in measuring mechanical properties of plant organ bindings. In addition, a method for the mechanical measurement of gravitropic bending forces in pea sprouts has been developed (Nakaso et al. 2020, pp 475-480) and provides us a chance to further understand gravitropic movement from a mechanical point of view.

We believe that this special issue will contribute not 
only to researchers working on plant biomechanics but also to researchers who have not considered plant biomechanics so far.

\section{References}

Abe Y, Meguriya K, Matsuzaki T, Sugiyama T, Yoshikawa HY, Morita MT, Toyota M (2020) Micromanipulation of amyloplasts with optical tweezers in Arabidopsis stems. Plant Biotechnol 37: 405-415

Akita E, Yalikun Y, Okano K, Yamasaki Y, Ohtani M, Tanaka Y, Demura T, Hosokawa Y (2020) In situ measurement of cell stiffness of Arabidopsis roots growing on a glass micropillar support by atomic force microscopy. Plant Biotechnol 37: 417-422

Bastien R, Legland D, Martin M, Fregosi L, Peaucelle A, Douady S, Moulia B, Höfte H (2016) KymoRod: A method for automated kinematic analysis of rod-shaped plant organs. Plant $J 88$ : 468-475

Beier MP, Tsugawa S, Demura T, Fujiwara T (2020) Root shape adaptation to mechanical stress derived from unidirectional vibrations in Populus nigra. Plant Biotechnol 37: 423-428

Burton RA, Gidley MJ, Fincher GB (2010) Heterogeneity in the chemistry, structure and function of plant cell walls. Nat Chem Biol 6: 724-732

Chelakkot R, Mahadevan L (2017) On the growth and form of shoots. J R Soc Interface 14: 20170001

Cosgrove DJ (2015) Plant expansins: Diversity and interactions with plant cell walls. Curr Opin Plant Biol 25: 162-172

Cosgrove DJ (2016) Catalysts of plant cell wall loosening. F1000 Res 5: 119

Geitmann A, Niklas K, Speck T (2019) Plant biomechanics in the 21st century. J Exp Bot 70: 3435-3438

Hagihara T, Toyota M (2020) Mechanical signaling in the sensitive plant Mimosa pudica L. Plants (Basel) 9: 587

Higaki T, Mizuno H (2020) Four-dimensional imaging with virtual reality to quantitatively explore jigsaw puzzle-like morphogenesis of Arabidopsis cotyledon pavement cells. Plant Biotechnol 37: 429-435

Ito K, Fukuoka K, Nishigaki N, Hara K, Yoshimi Y, Kuki H, Takahashi D, Tsumuraya Y, Kotake T (2020) Structural features conserved in subclass of type II arabinogalactan. Plant Biotechnol 37: 459-463

Kawakatsu Y, Sawai Y, Kurotani K, Shiratake K, Notaguchi M (2020) An in vitro grafting method to quantify mechanical forces of adhering tissues. Plant Biotechnol 37: 451-458

Kubo M, Udagawa M, Nishikubo N, Horiguchi G, Yamaguchi M, Ito J, Mimura T, Fukuda H, Demura T (2005) Transcription switches for protoxylem and metaxylem vessel formation. Genes Dev 19: 1855-1860

Kunieda T, Kishida K, Kawamura J, Demura T (2020) Influence of osmotic condition on secondary cell wall formation of xylem vessel cells induced by the master transcription factor VND7. Plant Biotechnol 37: 465-469

Lombard V, Golaconda Ramulu H, Drula E, Coutinho PM, Henrissat B (2014) The carbohydrate-active enzymes database (CAZy) in 2013. Nucleic Acids Res 42(D1): 490-495

Matsuyama K, Sunagawa N, Igarashi K (2020) Mutation of cysteine residues increases heterologous expression of peach expansin in the methylotrophic yeast Pichia pastoris. Plant Biotechnol 37: 397-403
Milani P, Gholamirad M, Traas J, Arnéodo A, Boudaoud A, Argoul F, Hamant O (2011) In vivo analysis of local wall stiffness at the shoot apical meristem in Arabidopsis using atomic force microscopy. Plant J 67: 1116-1123

Morita MT (2010) Directional gravity sensing in gravitropism. Annu Rev Plant Biol 61: 705-720

Moulia B, Der Loughian C, Bastien R, Martin O, Rodríguez M, Gourcilleau D, Barbacci A, Badel E, Franchel G, Lenne C, et al. (2011) Integrative mechanobiology of growth and architectural development in changing mechanical environments. In: Wojtaszek P (ed) Mechanical Integration of Plant Cells and Plants. Springer, Berlin, Heidelberg, pp 269-302

Nakai R, Azuma T, Nakaso Y, Sawa S, Demura T (2020) Development of a dynamic imaging method for gravitropism in pea sprouts using clinical magnetic resonance imaging system. Plant Biotechnol 37: 437-442

Nakaso Y, Arimoto S, Kawaguchi K, Muto T, Ueda H (2020) Mechanical measurement of gravitropic bending force in pea sprouts. Plant Biotechnol 37: 475-480

Nakata MT, Nakao M, Denda A, Onoda Y, Ueda H, Demura $\mathrm{T}$ (2020) Estimating the flexural rigidity of Arabidopsis inflorescence stems: Free-vibration test vs. three-point bending test. Plant Biotechnol 37: 471-474

Nakata MT, Takahara M, Sakamoto S, Yoshida K, Mitsuda N (2018) High-throughput analysis of Arabidopsis stem vibrations to identify mutants with altered mechanical properties. Front Plant Sci 9: 780

Niklas KJ, Spatz H-C (2000) Wind-induced stresses in cherry trees: Evidence against the hypothesis of constant stress levels. Trees (Berl) 14: 230-237

Notaguchi M, Kurotani K, Sato Y, Tabata R, Kawakatsu Y, Okayasu K, Sawai Y, Okada R, Asahina M, Ichihashi Y, et al. (2020) Cellcell adhesion in plant grafting is facilitated by $\beta$-1,4-glucanases. Science 369: 698-702

Peaucelle A (2014) AFM-based mapping of the elastic properties of cell walls: At tissue, cellular, and subcellular resolutions. J Vis Exp 89: e51317

Seifert GJ, Roberts K (2007) The biology of arabinogalactan proteins. Annu Rev Plant Biol 58: 137-161

Somerville C, Bauer S, Brininstool G, Facette M, Hamann T, Milne J, Osborne E, Paredez A, Persson S, Raab T, et al. (2004) Toward a systems approach to understanding plant cell walls. Science 306: 2206-2211

Speck O, Speck T (2019) An overview of bioinspired and biomimetic self-repairing materials. Biomimetics 4: 26

Tsugawa S, Kanda N, Nakamura M, Goh T, Ohtani M, Demura T (2020) Spatio-temporal kinematic analysis of shoot gravitropism in Arabidopsis thaliana. Plant Biotechnol 37: 443-450

Watanabe A, Rahman A, Nishitani K, Yamada R, Takahashi M, Tsugawa S, Demura T (2020) Laser micromarking technique in studying the negative gravitropism in pea stem. Plant Biotechnol 37: $485-488$

Yamaguchi M, Goué N, Igarashi H, Ohtani M, Nakano Y, Mortimer JC, Nishikubo N, Kubo M, Katayama Y, Kakegawa $\mathrm{K}$, et al. (2010) VASCULAR-RELATED NAC-DOMAIN6 and VASCULAR-RELATED NAC-DOMAIN7 effectively induce transdifferentiation into xylem vessel elements under control of an induction system. Plant Physiol 153: 906-914

Yoshida Y, Arita T, Otani J, Sawa S (2020) Visualization of Toyoura sand-grown plant roots by X-ray computer tomography. Plant Biotechnol 37: 481-484 